\title{
Digital matter as interdisciplinary commodity
}

\author{
Uwe Rieger \\ The University of Auckland, New Zealand \\ School of Architecture and Planning \\ u.rieger@auckland.ac.nz
}

\begin{abstract}
With the current exponential growth in the sector of Spatial Data Technology and Mixed Reality display devises we experience an increasing overlap of the physical and digital world. Next to making data spatially visible the attempt is to connect digital information with physical properties. Over the past years a number of research institutions have been laying the ground for these developments. In contemporary architecture architectural design the dominant application of data technology is connected to graphical presentation, form finding and digital fabrication.

The arc/sec Lab for Digital Spatial Operations at the University of Auckland takes a further step. The Lab explores concepts for a new condition of buildings and urban patterns in which digital information is connected with spatial appearance and linked to material properties. The approach focuses on the step beyond digital re-presentation and digital fabrication, where data is re-connected to the multi-sensory human perceptions and physical skills. The work at the Lab is conducted in a cross disciplinary design environment and based on experiential investigations. The arc/sec Lab utilizes large-scale interactive installations as the driving vehicle for the exploration and communication of new dimensions in architectural space. The experiments are aiming to make data "touchable" and to demonstrate real time responsive environments. In parallel they are the starting point for both the development of practice oriented applications and speculation on how our cities and buildings might change in the future.

The article gives an overview of the current experiments being undertaken at the arc/sec Lab. It discusses how digital technologies allow for innovation between the disciplines by introducing real time adaptive behaviours to our build environment and it speculates on the type of spaces we can construct when digital matter is used as a new dynamic building material.
\end{abstract}

Keywords: reactive architecture, digital matter, haptic-digital space, digital spatial technologies, arc/sec.

To cite this article:

Rieger, U. (20I7). Digital matter as interdisciplinary commodity. The Journal of Public Space, 2(3), Special Issue, I03-I I0, DOI: I0.5204/jps.v2i3.II9

This article has been peer-reviewed and accepted for publication in The Journal of Public Space. Please see the Editorial Policies under the 'About' section of the journal website for further information.

This work is licensed under a Creative Commons Attribution - Non Commercial 4.0 International License https://creativecommons.org/licenses/by-nc/4.0/ 
Architecture creates and utilises the space in which we live and operate. In our daily experience this space is characterised by the co-existence of the physical and the digital world. With the recent exponential developments and the availability of mixed reality devices and spatial data technology, both worlds are increasingly intertwining. With data as unified source of exchange, these technologies offer a common ground for interdisciplinary explorations and discoveries. The linkage between data, space and materiality allows allow to go beyond two dimensional screen interaction towards a towards responsive spatial environments. The newly released developer version of the Microsoft HoloLenses' and the highly anticipated Magic Leap ${ }^{2}$ glasses by Google are about to bring recent science fiction scenarios to life. This latest generation of augmented reality devices allow for gesturebased operation of spatial data, as in Tom Cruise's famous scene in Minority Report, ${ }^{3}$ from 2002, and the natural interaction with the coexistence of holographic and physical objects, as envisaged in the Iron Man movie series, ${ }^{4}$ from 2008, 2010 and 2013.

But as well as giving data a visual, local, spatial appearance through stereo displays, these new devices will allow for more, as integrated GPS, giros and scanning sensors identify location, the surrounding environment and objects. Effectively, this enables us to connect physical material with digital information, and the I:I calibration of the digital and physical world allows us to give data a tangible appearance.

An exemplary application of this strategy is the newly conceptualised entertainment centre the VOID, which is advertised to open globally by the end of this year: "Utilizing a layering of real-time interactive environments, and blending the real world with the digital, our participants are placed into our Hyper-Reality experiences."5The VOID project achieves this experience by overlaying the virtual world, displayed through Oculus Rift technology, with a physical build environment combined with pressure suites and kinetic machinery. The project is exemplary, as it demonstrates our desire to connect data to multisensory properties. This desire appears as a logical consequence given that human senses have evolved through the interaction with the physical world.

Hyper-Reality environments have been a fascinating and inspiring proposal in science fiction environments. A very early and well known example is the "Holodeck" in Star Trek's Next Generation. ${ }^{6}$ It is the vision of a computer-controlled room to generate holographic matter: usable, consumable and fully interactive as a responsive world with artificial life forms. Over the past years, institutions such as the Media Lab at the Massachusetts Institute of Technology (MIT) have been investigating the background of these speculative scenarios. Of specific interest here is the research of MIT's Media Lab into tangible data and radical atoms.

The Tangible Media Research group aims to elevate data from 2D screen-based graphical user interfaces (GUI), to 3D tangible user interfaces (TUI), and eventually to programmable matter. The director of the group, Professor Hiroshi Ishii, calls this type of matter radical

\footnotetext{
I "Microsoft Hololenses", accessed August 20, 2016. https://www.microsoft.com/microsoft-hololens/en-us.

2 “Magic Leap”. Accessed August 20, 2016. https://www.magicleap.com/.

${ }^{3}$ Minority Report, directed by Steven Spielberg (Amblin Entertainment, Cruise/Wagner Productions, 2002).

${ }^{4}$ Iron Man, directed by John Favreau (Marvel Studios, 2008); Iron Man 2, directed by John Favreau (Marvel Studios, 2010); Iron Man 3, directed by Shane Black (Marvel Studios, 2013).

5 “The VOID”. Accessed August 20, 2016. https://www.thevoid.com/.

${ }^{6}$ Star Trek -The Next Generation, produced by G. Roddenberry, and R. Berman (Paramount Domestic Television, 1984-1994).
} 
atoms ${ }^{7}$ and summarises the attempt in the vision "to seamlessly couple the worlds of bits and atoms by giving dynamic physical form to digital information and computation." Tangible Media Group has developed a series of projects to test these ideas; amongst them are the kinetic installation inFORM ${ }^{9}$ and the video animation Perfect Red. ${ }^{10}$

InFORM (figure I) demonstrates the concept of a TUI as a "Dynamic Shape Display that can render 3D content physically, so users can interact with digital information in a tangible way. InFORM can also interact with the physical world around it, for example moving objects on the table's surface. Remote participants in a video conference can be displayed physically, allowing for a strong sense of presence and the ability to interact physically at a distance."

The interaction conceptual video animation Perfect Red (figure 2) explains the idea of radical atoms. The video describes "a clay-like material pre-programmed to have many of the features of Computer-Aided Design (CAD) software. Perfect Red is a fictional material that can be sculpted like clay....and responds according to rules inspired by CAD operations, including snapping to primary geometries, Boolean operations, and parametric design. The idea of snapping to primary geometries such as sphere, cylinder, and cube was inspired by shape-memory alloys. ... Perfect Red is imagined as one of a number of new materials imbued with a complex set of responsive behaviors."

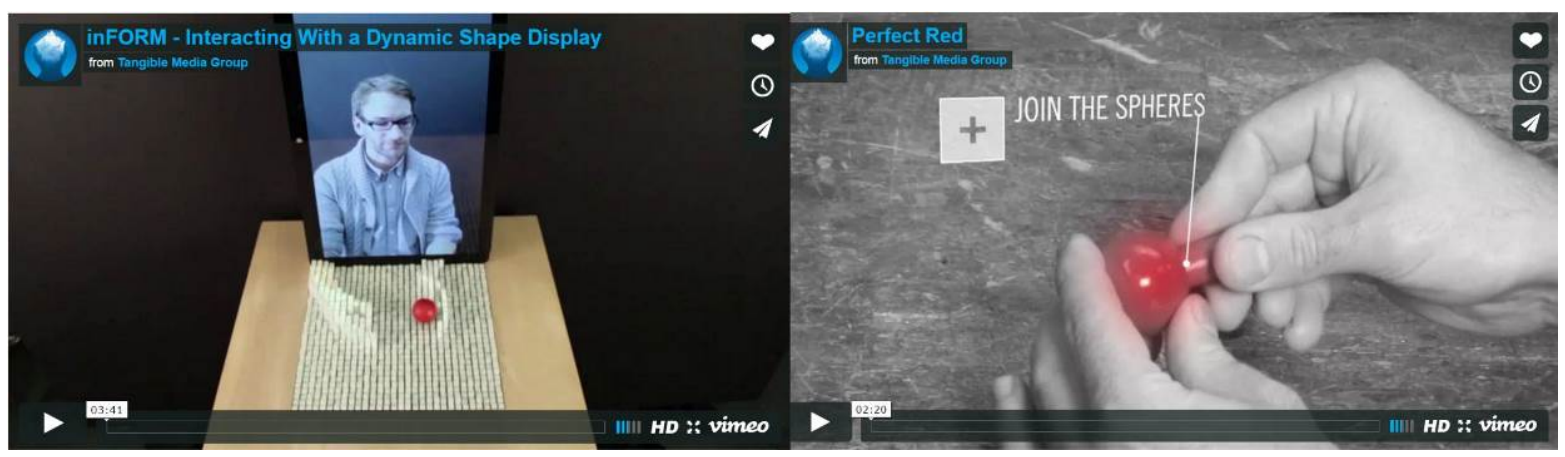

Figure I (left). inFORMhape shift display, MIT Media Lab, 2013.

Figure 2 (right). Perfect Red, digital matter, MIT Media Lab, 2012.

With the ability to create programmable space displayed through a new generation of wearable AR devices and the application of programmable materials, we are entering a new type of living environment. The traditional separation of computer and user is being dissolved, towards a "perspective that acknowledges how people, computational materials,

\footnotetext{
${ }^{7}$ Hiroshi Ishii, Dávid Lakatos, Leonardo Bonanni, and Jean-Baptiste Labrune, "Radical Atoms: Beyond Tangible Bits, Toward Transformable Materials", Interactions I9, n. I (20I2): 38-5I.

8 “Vision Statement", MIT Media Lab, Tangible Media Group. Accessed August 20, 2016.

https://www.media.mit.edu/research/groups/tangible-media.

${ }^{9}$ InFORM, MIT Media Lab, Tangible Media Group. Accessed August 20, 2016. http://tangible.media.mit.edu/project/inform/.

${ }^{10}$ Leonardo Bonanni, Hiroshi Ishii, Austin Lee, Paula Aguilera, and Jonathan Williams, Perfect Red, MIT Media Lab, Tangible Media Group. Accessed August 20, 2016. http://tangible.media.mit.edu/project/perfect-red/. II “InFORM," http://tangible.media.mit.edu/project/inform/.

${ }^{12}$ Bonanni, Ishii, Lee, Aguilera, and Williams, Perfect Red.
} 
and even traditionally non-computational materials are coming together as a whole, forming our experiences in and of the world."13 This new view of human computer interaction $(\mathrm{HCl})$ was discussed and formulated in a panel titled "Material InteractionsFrom Atoms and Bits to Entangled Practices" at the ACM CHI Conference in 20I2. The different viewpoints taken had two aspects in common. The connection of $\mathrm{HCl}$ to the material-based design disciplines and the focus on the user experience: "It also simultaneously prompts us to conceptualize computers not as black boxes, but as yet another design material operating in concert with other physical materials-again, with a focus on what these material assemblages can enable in terms of new user experiences and new practices." 14

Digital technologies allow designers and engineers to introduce dynamic behaviours and to create adaptive schematics, which expand conventional practice and the understanding of architecture as a static and rigid element. In contemporary architectural design, data technology is still connected to graphical presentation, form finding and digital fabrication. While real time, responsive relationships are normal attributes of the digital realm, the question still remains as to what type of environments we can generate when data appears to be physical in space and what type of spaces we can build when data is used as an interactive construction material, complementing traditional materials like stone, concrete, glass etc. The designer and film-maker Keiichi Matsuda, with his work, attempts to give some answers to the implications of emerging technologies for human perception and the built environment. His latest online video Hyper-Reality ${ }^{15}$ documents the overwhelming opportunities for design, the challenges of interaction and threats of triviality. But it also makes clear how important it will become for human wellbeing to start developing design principles for haptic-digital environments.

It is within this context that we have conceived the arc/sec Lab for Spatial Digital Operations at the School of Architecture and Planning at the University of Auckland in 2015. The lab explores concepts for new conditions of buildings and urban patterns, in which digital information will be given spatial appearance and physical form. The approach focuses on the next step beyond 2D graphical presentation and 3D digital fabrication, where data operates with tangible properties. While multinational companies are rapidly developing new spatial digital technologies, the objective of the arc/sec lab is to apply technology and explore haptic-digital space specifically from an architectural design perspective.

Based on the recent experience with New Zealand's inter-school project Studio[]Christchurch, the Lab has adapted its three key principles: "teamwork, crossdisciplinary collaborations and collective learning." body of work, the investigations are goal oriented, applied and long term. The purpose of our research is to expand architectural understanding of real time reactive environments.

\footnotetext{
${ }^{13}$ Mikael Wiberg, Hiroshi Ishii, Paul Dourish, Anna Vallgårda, Tobie Kerridge, Petra Sundström, Daniela Rosner, and Mark Rolston, “Materiality Matters_Experience Materials,” Interactions 20, n. 2 (2013): 54-57. doi: | 0. I | 45/2427076.2427087

${ }^{14}$ Wiberg, Ishii, Dourish, Vallgårda, Kerridge, Sundström, Rosner, and Rolston, "Materiality Matters-

Experience Materials”, Interactions 20, 2 , 20I3, 54-57, DOI: I0.I I45/2427076.2427087

${ }^{15}$ Keiichi Matsuda, Hyper Reality. Accessed August 20, 2016. http://hyper-reality.col.

${ }^{16}$ Uwe Rieger and Camia Young, "Design Through Collective Learning," in Studio Futures: Changing Trajectories in Architectural Education, ed. Donald Bates, Vivian Mitsogianni, and Diego Ramírez-Lovering (Melbourne: Uro Publications, 20I5), 59-76.
} 
We are interested in user interaction and navigation in haptic-digital space by adding dynamic properties to the traditional perception of static architecture.

The underlying research question of the arc/sec projects is: What are the functional, programmatic and aesthetic design parameters for interactive environments that use digital matter as a new source of construction material? The arc/sec lab utilises large-scale interactive installations as the driving vehicle for the exploration and communication of time-based architectural design. We are developing prototypical space and immersive environments for two reasons, to demonstrate new ideas of reactive architecture and to learn how the user navigates and understands haptic-digital space. While it is not yet technically possible to generate free spatial holograms or free-forming digital matter, it is , within limits, possible to construct a sense of digital matter by using traditional 3D projection strategies in combination with latest scanning technology and real time rendering software.

The following three arc/sec projects, which are using different methods to generate a real time responsive environment and the sensation of tactile data space, demonstrate the potential of the research.

HyperSpace (figure 3) creates an augmented (hyper) reality world by illuminating haze particles. Large 3D constructions of light appear interactively in space. They are visible without the need of any additional devices such as goggles, screens, helmets etc. The setup combines a live-render gaming engine with a motion-capture system and multiple laser projectors. Virtual world and physical environment are calibrated one to one. The outcome is a full 360 degree haptic-digital space, accurately defined in all dimensions, interactive and inhabitable (figure 4). The installation reacts to body moment, body functions, sound and other environmental factors in order to generate architectural space.

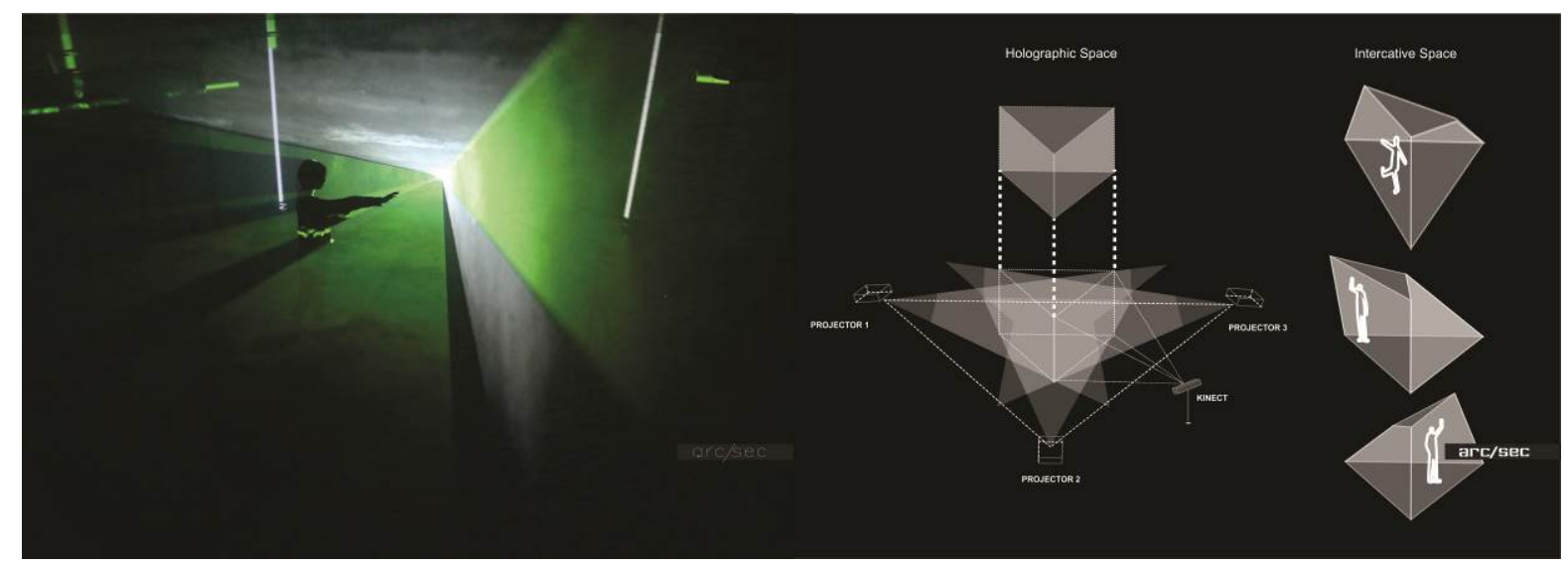

Figure 3 (left). HyperSpace, illuminated haze particles.

Figure 4 (right). HyperSpace interactive holographic volumes (drawing Yinan Liu).

The technical background was developed with a team of postgraduate students at the $\mathrm{arc} / \mathrm{sec}$ Lab in 2015. The expanded version, SINGULARITY, was explored as a master's thesis project by Yinan Liu and Ying Miao in 2016. The project runs in collaboration with dance studies and the Sonic Arts Department at the University of Auckland. A public 40 minute test performance was presented at the Kenneth Myer Centre in Auckland on June 4, 2016. The final 70 minute show, featuring national and international dance and sound artists, is 
scheduled for November 2 in the renowned Q-Theatre in Auckland. As an architectural project it explores $\mathrm{HCl}$ with digital constructions and digital matter.

The LightScale II installation (figure 5,6) generates a tactile experience of 3D data through projections onto multiple layered gauze surfaces. The kinetic structure consists of a 20metre long carbon mast construction, surrounded by multiple layers of black mesh. The construction is mounted asymmetrically on a single pin-point support. On a touch of the visitor this allows the construction to oscillate in space, freely and with almost no friction. A tracking system recognises the position and movement of the LightScale. A live-render program overlays the physical construction with projected digital information. The installation combines three types of data sets: Firstly, place bound information which only appears in specific positions; secondly, tracked data following the kinetically moving object; and thirdly, responsive data which corresponds to the user interaction with the LightScale. Like a giant creature, it floats through a virtual ocean materialising environments, stories and user interactions. The project has its roots in a design by kunst und techink ${ }^{17}$ (R. Hartl, M. Janekovic, U. Rieger, H. Schroeder) in Berlin in end 90's. Equipped with latest digital spatial technologies LightScale II now advances towards an responsive navigation tool that creates haptic-digital constructions and materializes spatial narratives.

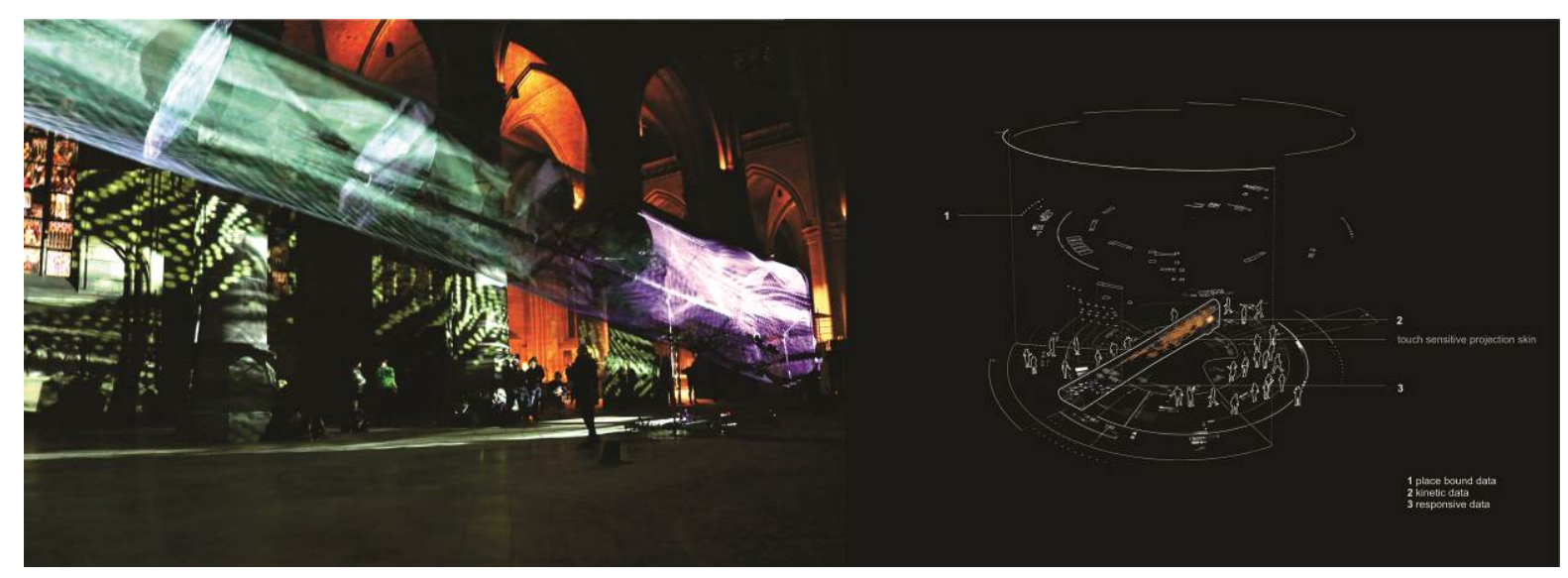

Figure 5 (left). LightScale II, 20 m long carbon construction at the New Cathedral Linz Austria. Figure 6 (right). LightScale II, physical construction and augmented data sets (drawing Yiqiu Hong).

Anaglyph is a 3D display system (figure 7,8 ) that puts multi-layered digital information into a spatial order. The installation augments a physical setup with digital information by using the well-known red/cyan stereo projection system to generate virtual 3D constructions. The prototype installation was tested with a team of 3rd year architecture students in the first half of 2016. It consists of a $4 \mathrm{~m} \times 8 \mathrm{~m}$ curved screen, a simple Kinect camera and 4 laser projectors to create an interactive and immersive environment that allows a display of data in the form of text, images, drawings and sound. The unique features of the AnaGlyph installation are its sculptural haptic-digital quality and its spatial architectural navigation system.

\footnotetext{
${ }^{17}$ Angelika Schnell, "Kunst und Technik eV.”, in Young German Architects 2, (Germany: Birkhaeuser Verlag 2000), 88-96.
} 
In collaboration with the South Pacific Department at the Auckland War Memorial Museum, an applied version is currently under development. The aim is to make the museum's extensive data archive on the Pacific Islands, their history and culture, accessible with a specific focus on migration and maritime travel. The new setup will use an advanced tracking system to pick up user interaction with displayed physical navigation tools and artefacts. The setup allows access to information as a new haptic-digital museum experience. The main architectural investigation of this project is the spatial organisation of data and how this data can be combined with physical objects and physical navigation tools

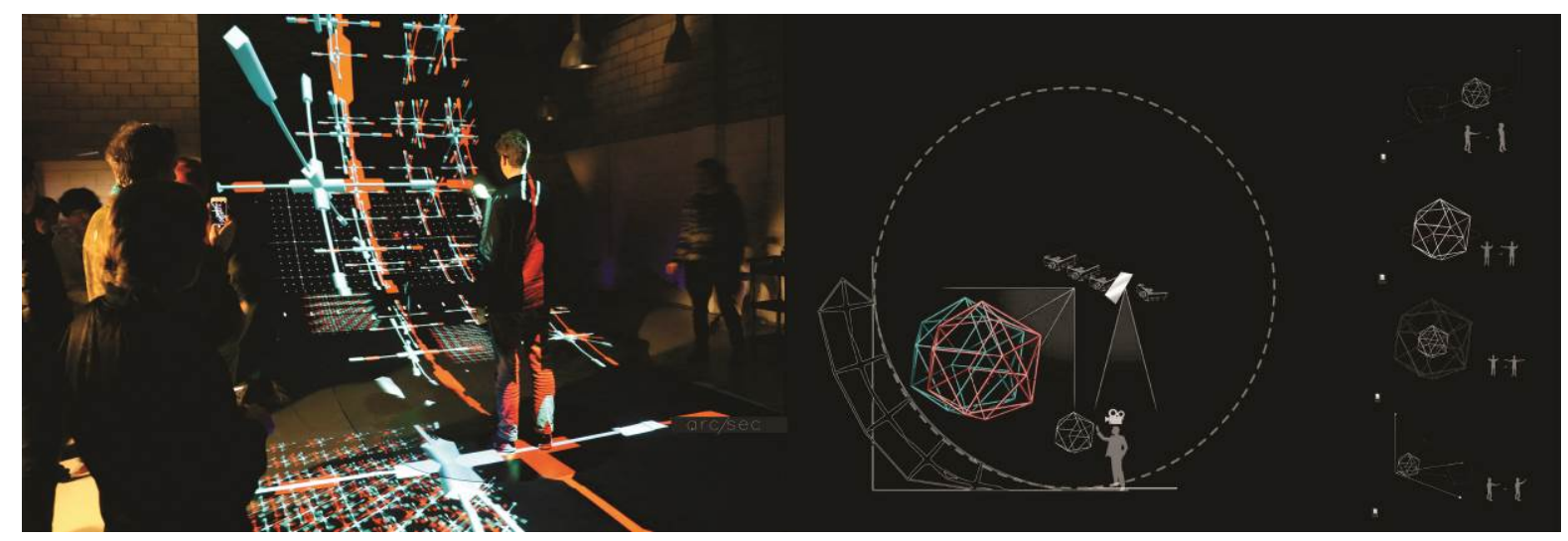

Figure 7 (left). AnaGlyph, 3D digital constructions generated through red/cyan projections Figure 8 (right). AnaGlyph, digital construction overlayed with physical navigation tools.

The principle of projecting onto layered surfaces to generate spatial appearances is well known. It is used for both, to generate spectacular entertainment shows and refined artistic projects. Examples for this are the mesh projections by Nonotak Studio ${ }^{18}$, such as Daydream V2 I, and Anthony McCall's Solid Light Works ${ }^{19}$, that work with illuminating fog particles. What makes the arc/sec projects distinct is the I:I calibration of an interlinked virtual world with a physical setup. The space generated is not a 2D animation creating a 3D effect, rather it is a precisely calculated environment, defined in three dimensions by using a spatial sensors and an array of data projectors. From the beginning the design process integrates physical and digital reality and the outcome is a fusion of both worlds, creating a new form of haptic-digital materiality and responsive architecture. Research and experiments at the arc/sec lab are not to aiming for a new technical invention, but to discover the unknown through making new connections between the disciplinary knowledge. In our recent collaborations with dance, music, engineering and medical science, it became evident that data is the shared ground and the common source for interdisciplinary operation. Data is interchangeable; it may appear as music, images, calculations, text, programs, sensor inputs and outputs, etc. Spatial-digital technologies offer a link between the disciplines and are the shared tools with which to process data as raw material. As the authors of the article "Materiality Matters-Experience Materials" conclude, "acknowledging the computational as a material is indeed a radical shift in

\footnotetext{
${ }_{18}$ Nonotak Studio, Daydream V.2. Accessed July 19, 2017. http://www.nonotak.com/_DAYDREAM-V-2

${ }^{19}$ Anthony McCall, Current and Upcoming". Accessed July 19, 2017. http://www.anthonymccall.com/
} 
perspective in $\mathrm{HCl}-\mathrm{a}$ shift from how IT is applied toward a focus on the material character of IT." 20

With data acquiring spatial-visual appearance, sensory properties and physical presence we will not only need to redefine $\mathrm{HCl}$, but consequently our interaction with the built environment. Architecture as a spatially operating design discipline, with its ability to coordinate multidisciplinary construction processes, is hereby in an ideal position to facilitate this journey of new discoveries.

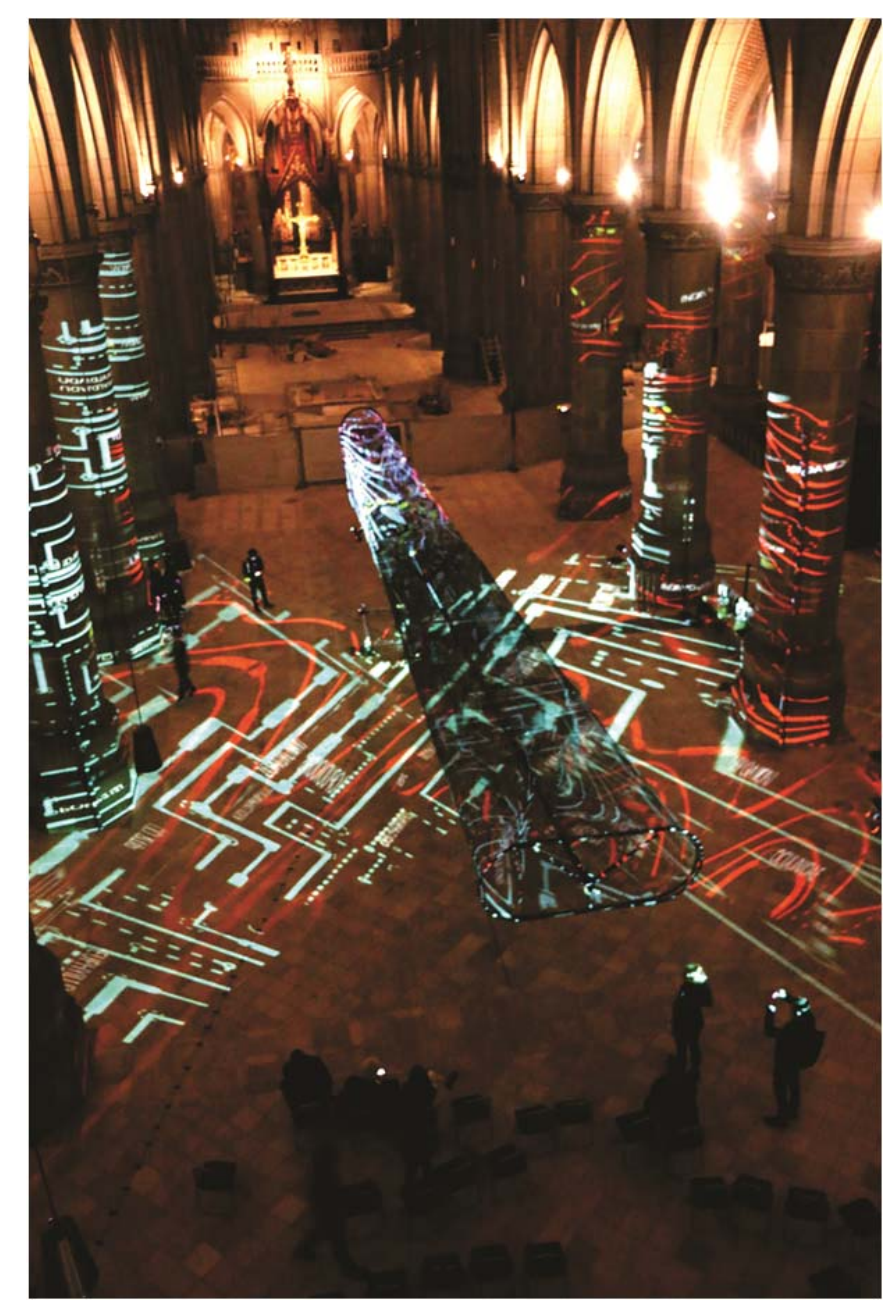

Figure 9. LightScale II at the Ars Electronica Festival, New Cathedral Linz, Austria, 2017.

\footnotetext{
${ }^{20}$ Wiberg, Ishii, Dourish, Vallgårda, Kerridge, Sundström, Rosner, and Rolston, "Materiality MattersExperience Materials", Interactions 20, 2 , 20I3, 54-57, DOI: 10.1 I45/2427076.2427087
}

I I 0 | The Journal of Public Space, 2(3), 2017 | Special Issue | ISSN 2206-9658

(c) Queensland University of Technology 